\title{
Early Pain, Narcotic Use, and Functional Recovery in Aseptic Revision Total Knee Arthroplasty Performed with Morphine Spinal Analgesia versus Fentanyl Spinal Analgesia with Adductor Canal Block
}

\author{
Justin Rice BA ${ }^{1}$, Amer Mohiuddin BS ${ }^{1}$, Trent Nielson ${ }^{2}$, \\ Mary Ziemba-Davis $\mathrm{BA}^{3}$, R. Michael Meneghini $\mathrm{MD}^{1,3}$ \\ ${ }^{1}$ Indiana University School of Medicine, Indianapolis, IN \\ ${ }^{2}$ Indiana University, Bloomington, IN \\ ${ }^{3}$ IU Health Physicians, Orthopedics \& Sports Medicine, Fishers, IN
}

Background and Hypothesis: There has been a trend away from intrathecal morphine for perioperative pain control during and after total knee arthroplasty (TKA) and it has been replaced by shorter-acting non-morphine spinals combined with adductor canal block (ACB) regional analgesia. The purpose of this study is to compare pain control and side-effects between morphine intrathecal alone and fentanyl spinal with ACB in revision TKA.

Project Methods: Prospectively collected data on 167 consecutive aseptic revision TKAs performed by a single surgeon between 2010 and 2017 were retrospectively reviewed. Inpatient pain, narcotic use, functional milestones, complications, and four-month patient reported outcomes were compiled for the two cohorts (110 fentanyl/ACB and 57 morphine cases).

Results: Average age $(p=0.66)$ and BMI $(p=0.542)$ were equivalent in the two cohorts $(p=0.542)$. Fentanyl/ACB patients gained more range-of-motion (ROM) by 4-month follow-up ( $p=0.051)$ than morphine patients. However, fentanyl/ACB patients had higher average pain scores $(p=0.006)$, consumed opioids earlier $(p<0.001)$, and consumed more opioids $(p<0.001)$ on postoperative day one. Morphine analgesia was associated with more nausea/vomiting $(p=0.001)$ and pruritus $(p=0.00001)$.

Conclusion and Potential Impact: Morphine intrathecal patients reported less inpatient pain and consumed less opioid, which most likely reflects morphine's longer half-life. Improved 4-month ROM in the fentanyl/ACB group may reflect the quadriceps-sparing nature of ACBs. It is also possible that the operative field in revision TKA extends beyond the sensory protection of ACB. Further research is required to elucidate the relative impact of $A C B s$ on primary vs. revision procedures. 